\title{
Evaluation of Two Commercially-Available Lubricants by Means of Ring Test to AA 6061 F Aluminum Alloys
}

\author{
Rogério Alves Oliveira ${ }^{\mathrm{a}}$, Leopoldo Alguero Koller $^{\mathrm{b}}$, Lirio Schaeffer \\ ${ }^{a}$ MSc Engenheiro Mecânico, Gerdau Aços Finos Piratini \\ Avenida Getúlio Vargas, 3200, 96745-000 Charqueadas - RS, Brazil \\ ${ }^{\mathrm{b}}$ Engenheiro Mecânico, Krupp Metalúrgica Campo Limpo \\ Avenida Alfried Krupp, 1050, 13231-900 Campo Limpo Paulista - SP, Brazil \\ ${ }^{c}$ Prof. Dr.-Ing, Laboratório de Transformação Mecânica - EEUFRGS \\ Avenida Bento Gonçalves, 9500, 91501-970 Porto Alegre - RS, Brazil
}

Received: February 20, 2003; Revised: July 26, 2003

\begin{abstract}
This paper shows friction results obtained through compression ring tests for two commercially-available lubricants (i.e., Oildag and Deltaforge) applied in the hot forging of aluminum alloys. The experiments were performed with AA $6061 \mathrm{~F}$ aluminum alloy samples with the goal of observing the behavior of each lubricant in several conditions of temperature $(200,300$, and $\left.450{ }^{\circ} \mathrm{C}\right)$, strain rates $\left(1,10\right.$ and $\left.50 \mathrm{~s}^{-1}\right)$, and strain $(25,50$ and $75 \%)$. The friction coefficients for each lubrication condition were established by means of calibration curves determined from finite element calculations with the use of the PEP/Larstran software package. An Analysis-of-Variance approach for the ring's internal diameter was followed in order to evaluate the performance of the lubricants. The results indicated that the Oildag lubricant presents a better stability as well as lower friction coefficients than the Deltaforge lubricant for the range of conditions tested, which translates into a better friction condition at die-part interface.
\end{abstract}

Keywords: friction, lubricants, aluminum alloys, hot forging

\section{Introdução}

Os lubrificantes utilizados em forjamento tem a sua maior aplicabilidade na necessidade de redução das cargas de forjamento e principalmente na diminuição dos desgaste da matriz. Para o forjamento de ligas de alumínio os fabricantes recomendam lubrificantes constituídos de uma solução a base de grafite + água ou grafite + óleo que para facilitar a sua aplicação são diluídos em proporções adequadas para o processo em que são utilizados.

À medida que são aprimorados os processos de forjamento, principalmente a quente (isotérmico ou não) onde o material sofre grandes deformações e exige-se mais da peça final quanto à precisão dimensional, aumenta a importância do conhecimento do atrito e de seu controle através de uma lubrificação adequada.

O conhecimento do atrito pode proporcionar importantes subsídios no desenvolvimento do processo de forjamento e propiciar benefícios quanto ao custo final do forjado e sua competitividade comercial.

Até mesmo os métodos mais avançados, baseados em modelos matemáticos como a teoria das tiras, do limite superior, elementos finitos, entre outros exigem que se conheçam as variáveis que regem o processo, dentre as quais o atrito se destaca fortemente para a obtenção de resultados coerentes e satisfatórios.

A importância de uma adequada condição de atrito peçamatriz ganha ainda mais destaque quando se deseja forjar novas ligas, peças complexas ou realizar forjamento de precisão, pois o atrito excessivo pode dificultar ou até mesmo impedir a realização do processo de conformação.

A idéia básica de amenizar os efeitos do atrito consiste em estabelecer um filme com baixa tensão de cisalhamento na interface peça-matriz para reduzir as tensões que se opõe ao movimento relativo nesta interface.

A lubrificação nos processos de conformação ${ }^{1}$ pode ser classificada nos seguintes tipos básicos:

a) condições fluidas ou hidrodinâmicas: quando existe

*e-mail: rogerio.oliveira@gerdau.com.br 
um filme de lubrificante suficientemente espesso para separar as duas superfícies;

b) condições de contorno ou de fronteira: quando existe um filme de espessura molecular separando as superfícies e zonas onde há contato entre rugosidades;

c) condições de lubrificação mista: que é a combinação das anteriores;

d) condição seca: quando não há lubrificante na interface.

Os lubrificantes, de uma maneira geral, devem apresentar as seguintes características e desempenhar algumas funções que em geral podem ser²:

a) Agir como um agente na prevenção da aderência e soldagem da peça na matriz;

b) Possuir boas propriedades de isolamento evitando perdas de calor, especialmente no forjamento a quente;

c) Ser inerte para prevenir ou reduzir reações entre a matriz e a peça nas temperaturas de forjamento empregadas;

d) Não ser abrasivo, reduzindo a erosão na superfície da matriz evitando o excessivo desgaste;

e) Ser livre de componentes poluidores ou venenos, não produzindo gases que ponham em risco a saúde dos operadores ou o meio ambiente;

f) Ser facilmente aplicável e removível da peça e da matriz;

g) Ser comercialmente disponível a um custo acessível.

Em geral, é difícil que sejam atendidas todas estas exigências e não existe método que possa avaliar todas elas ${ }^{2}$. Entretanto, pode-se quantificar a condição de lubricidade de um determinado lubrificante sob determinadas condições, de forma que estes valores obtidos sejam empregados na otimização do processo de conformação aplicado.

A avaliação de lubrificantes pela quantificação do atrito associado é uma das formas de se comparar e determinar o valor de um lubrificante.

As questões relacionadas à emissão de gases tóxicos e o perigo que os mesmos oferecem ao pessoal de fábrica devem ser pesados adequadamente inclusive atendendo os procedimentos próprios de cada empresa quanto ao recebimento, manuseio, utilização e o descarte do resíduo originado $^{2}$.

Entretanto, é importante a análise do comportamento destes lubrificantes no que concerne às variações nos parâmetros tecnológicos típicos de um processo de conformação a quente.

Sabe-se que a temperatura determina a natureza do lubrificante a ser utilizado, principalmente pelos seus efeitos na viscosidade e na formação de óxidos na superfície do material, assim como a velocidade de deformação e o grau de deformação podem apresentar variações que definem a condição mais econômica ou a de melhor qualidade no processo produtivo.
Portanto, é mandatório que os lubrificantes permitam obter uma condição de baixo atrito independente destes parâmetros típicos dos processos de conformação a quente de forma a propiciar um número menor de fatores controláveis em um processo produtivo.

Dentre os fatores que influenciam o atrito, destacam-se ${ }^{1}$ :

a) Viscosidade do lubrificante e a velocidade de deformação;

b) Temperatura;

c) Rugosidade da ferramenta;

d) Deformação;

\section{Fundamentação Teórica}

$\mathrm{O}$ atrito na interface de dois materiais pode ser quantificado em termos de um fator ou coeficiente. A tensão de cisalhamento de atrito, $\tau$, é normalmente expressa ${ }^{2,3}$, conforme a Lei do Atrito de Coulomb por

$$
\tau=\sigma_{n} \mu
$$

A Equação (1) indica que a tensão de cisalhamento é proporcional a $\sigma_{\mathrm{n}}$, componente da tensão normal na interface matriz/material e $\mu$ é o "coeficiente" de atrito. A tensão de cisalhamento do atrito, também pode ser expressa por ${ }^{2,3}$

$$
\tau=\frac{m}{\sqrt{3}} k_{\mathbf{f}}
$$

A Eq. 2 expressa $\tau$ em função da tensão de escoamento do material, $\mathrm{K}_{\mathrm{f}}$ e do "fator de cisalhamento" do atrito $\mathrm{m}$.

A determinação experimental do coeficiente de atrito $(\mu)$ ou do fator de cisalhamento do atrito $(\mathrm{m})$ é realizada com ajuda do chamado "Teste do Anel", que consiste na compressão de um anel com as dimensões do diâmetro externo, diâmetro interno e espessura inicialmente conhecidas.

Uma condição de atrito alto faz o diâmetro interno diminuir a medida que o anel é deformado e uma condição de atrito baixo faz o diâmetro interno aumentar em relação ao raio neutro à medida que o anel é deformado.

A Fig. 1 mostra anéis deformados e a respectiva condição de atrito interfacial entre matriz e peça, ou seja, (a) anel inicial, (b) anel deformado com atrito alto e (c) anel deformado com atrito baixo.

O "Teste do Anel" apresenta uma vantagem quando aplicado ao estudo do atrito que é o fato de não ser considerada a força necessária para deformar o anel. É necessário que a máquina de ensaios permita deformar e seja possível controlar esta deformação para que o aumento ou a diminuição relativa do diâmetro interno do anel seja comparado com a diminuição relativa da espessura do anel. 


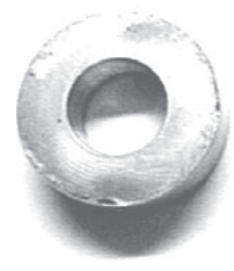

a)

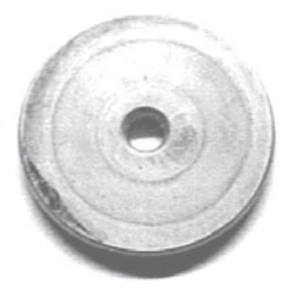

b)

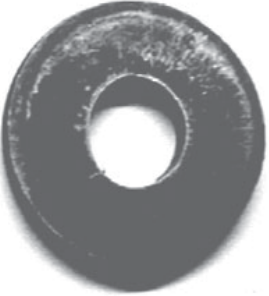

c)
Figura 1. Anéis deformados no ensaio de compressão.

Para se obter o valor do atrito, a redução percentual do raio interno em relação à redução percentual de espessura deve ser comparada com "curvas de calibração", traçadas para determinados valores de atrito e obtidas através de análises teóricas que simulam o fluxo de material durante a compressão e descrevam o abaulamento do corpo de prova para determinados valores de atrito.

Avitzur (1968) propôs equações analíticas ${ }^{4}$ para a compressão de um anel considerando os efeitos do atrito, através da análise pelo Método do Limite Superior/Inferior, onde as seguintes hipóteses são aplicadas:

a) A deformação é homogênea e ocorre na direção axial (não ocorre o embarrilamento);

b) A distribuição das forças de atrito de contato entre as superfícies é uniforme;

c) O material apresenta comportamento rígido-plástico perfeito.

Os programas de simulação numérica pelo método dos elementos finitos permitem obter as dimensões teóricas do corpo de prova deformado sob cada condição de atrito e assim é possível traçar as "curvas teóricas de calibração".

Marcon et al. (2000) apresentam um método que permite determinar no mesmo ensaio de compressão a curva tensão-deformação e o coeficiente de atrito $(\mu)$, utilizando o "Teste do Anel". Na Fig. 2 é mostrada a curva de calibração obtida pelo código "METAFOR", originalmente desenvolvido na Universidade de Liège na Bélgica, comparada com a solução aproximada de Avitzur (1968).

Kopp e Wiegels (1998) descrevem um modelo ${ }^{6}$ de ensaio do tubo cônico proposto por Philipp (1993), onde as curvas de calibração também são obtidas por simulação numérica (Elementos Finitos). A Fig. 3 mostra o corpo de prova do ensaio do tubo cônico e na Fig. 4 a curva de calibração obtida por simulação numérica para este modelo.

\section{Procedimento experimental}

Foram preparados corpos de prova de liga de alumínio ABNT $6061 \mathrm{~F}$ com as dimensões conforme mostrado na

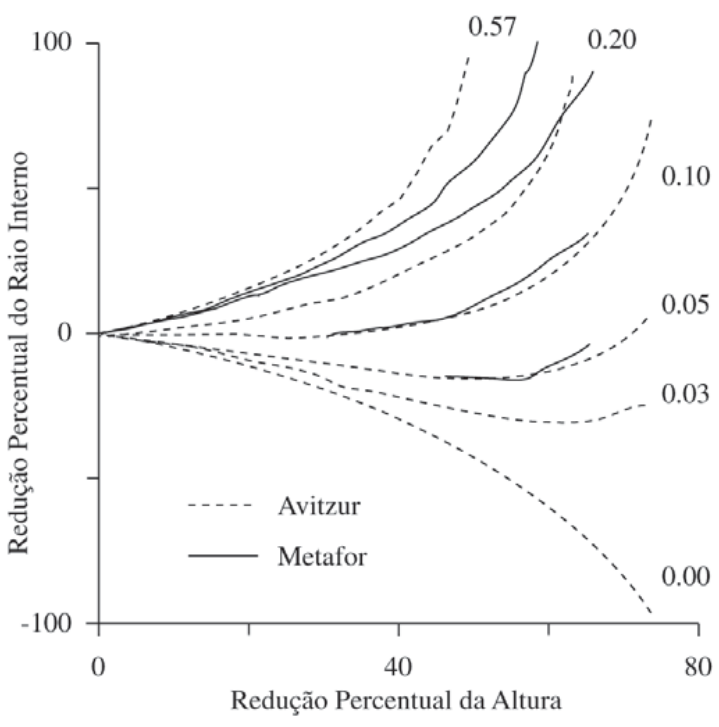

Figura 2. Comparação entre a solução numérica e a analítica ${ }^{5}$.
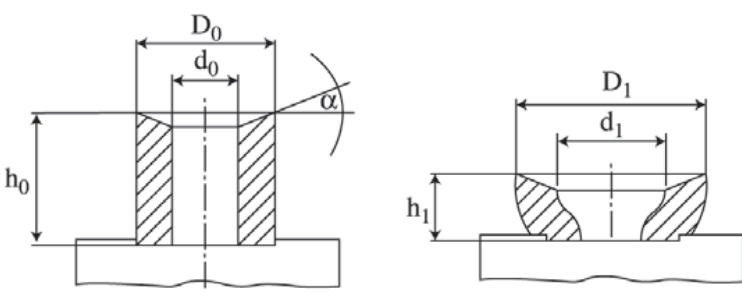

Figura 3. Corpo de prova para ensaio do tubo cônico ${ }^{6}$.

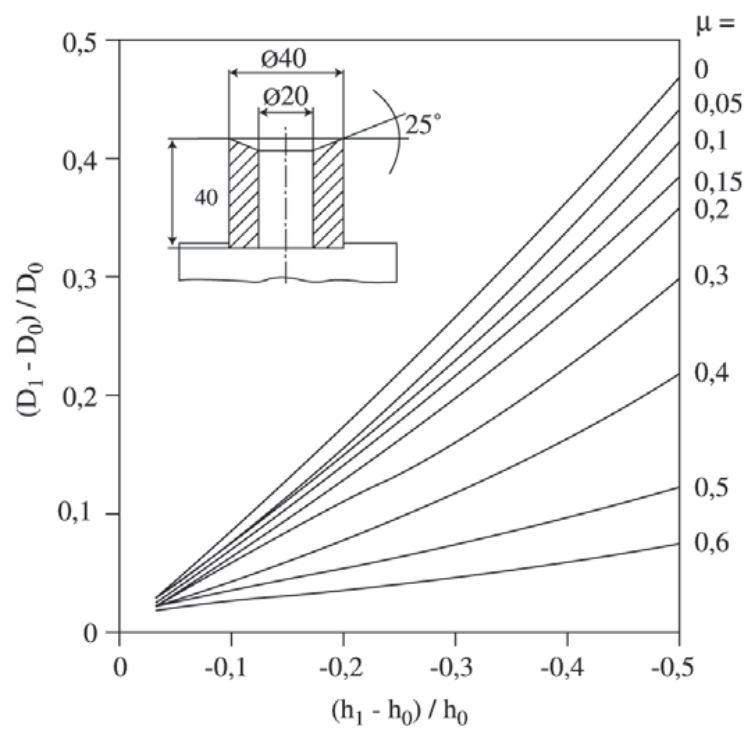

Figura 4. Curvas de calibração anel e tubo cônico ${ }^{6}$. 


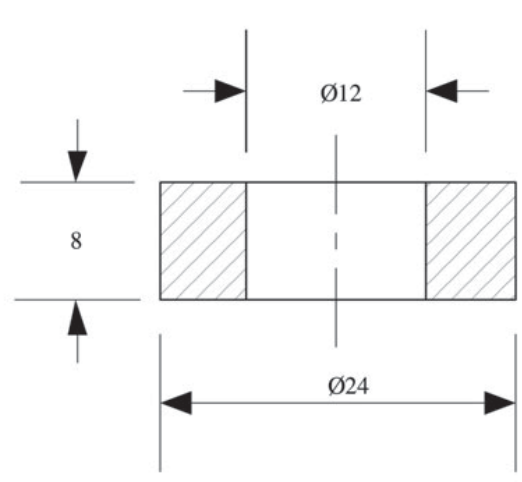

Figura 5. Dimensões do corpo de prova para o ensaio do anel.

Fig. 5, determinando um anel de proporções 6:3:2 em número suficiente para ensaiar três condições de contato: a) sem lubrificante b) lubrificante "Deltaforge 31" e c) lubrificante "Oildag", ambos da empresa Acheson do Brasil.
Os ensaios foram realizados em uma máquina servohidráulica controlada por um microcomputador e dotada de um forno de resistência elétrica ao redor dos punções. Esta construção permite manter o ensaio na condição isotérmica para as temperaturas 200,300 e $450^{\circ} \mathrm{C}$ com uma tolerância de $\pm 5^{\circ} \mathrm{C}$. A Fig. 6 mostra o controle da máquina.

$\mathrm{O}$ punção superior está ligado a uma célula de carga calibrada que envia o sinal de força para o sistema de aquisição de dados. O punção inferior realiza o movimento de compressão cujo sinal é medido por um transdutor potenciométrico e enviado para o mesmo sistema de aquisição de dados da célula de carga.

O movimento do punção inferior é controlado por um programa desenvolvido no Laboratório de Transformação Mecânica (LdTM), especialmente para a máquina de ensaios, o qual permite monitorar a velocidade de deformação constante durante o ensaio.

A máquina foi programada para obter anéis com deformações de $0,25,0,50$ e $0,75 \%$, nas velocidades de defor-

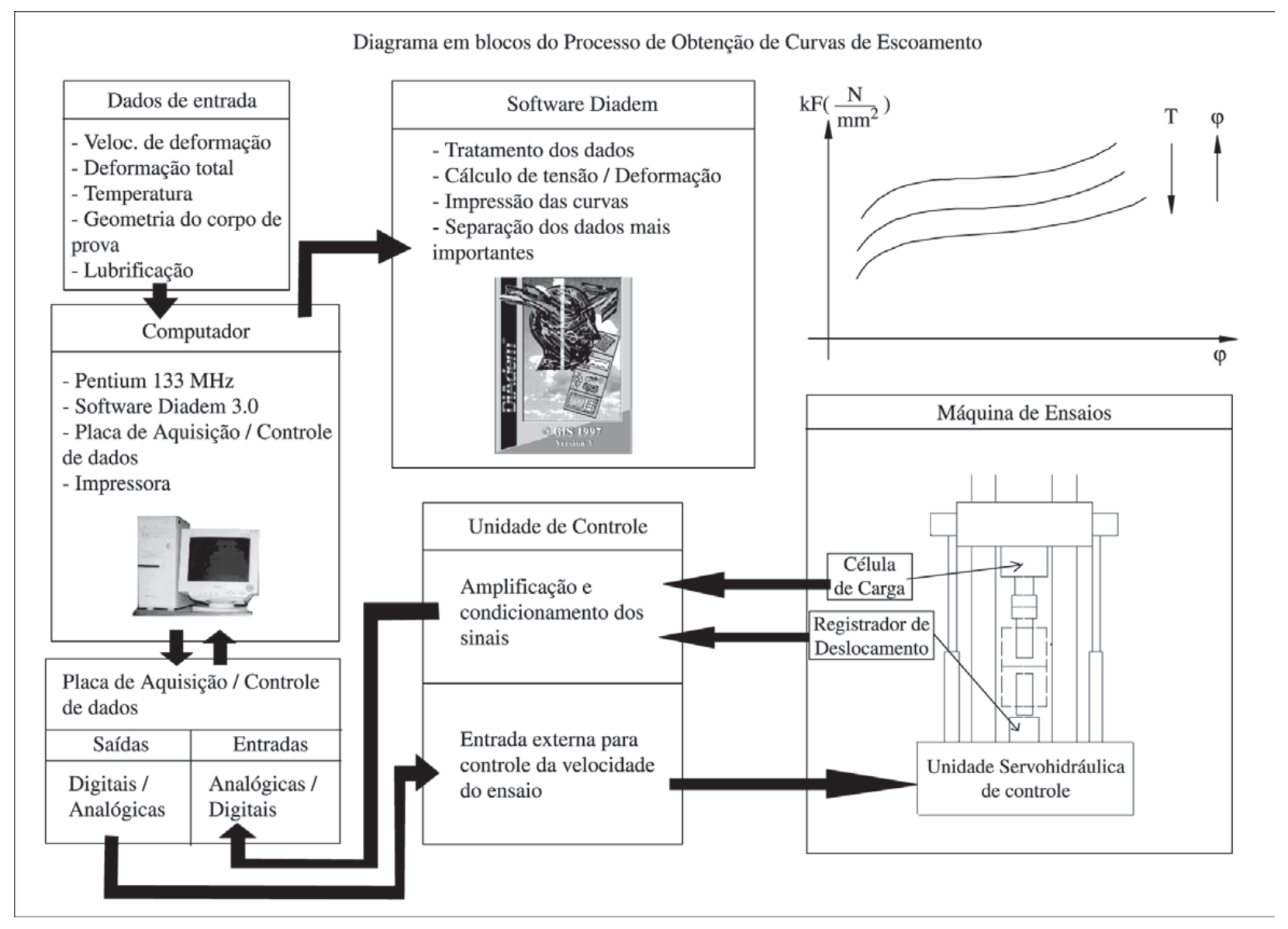

Figura 6. Esquema de controle da máquina de ensaios. 
mação de 1,10 e $50 \mathrm{~s}^{-1}$. Os corpos de prova atingiram deformações maiores que os valores programados, sem, contudo, comprometer a validade dos resultados. As curvas de calibração foram obtidas através da simulação numérica por elementos finitos no programa LARSTRAN (UT Aachen - Alemanha) da deformação de um anel com as mesmas dimensões da Fig. 5.

$\mathrm{Na}$ análise estatística, foram considerados os seguintes níveis de cada parâmetro, para cada tipo de lubrificante ("Deltaforge 31" e "Oildag"):

(i) Temperatura: 3 níveis $\left(200,300\right.$ e $\left.450{ }^{\circ} \mathrm{C}\right)$;

(ii) Velocidade de deformação: 3 níveis $\left(1,10\right.$ e $\left.50 \mathrm{~s}^{-1}\right)$;

(iii) Deformação Percentual na Altura: 3 níveis $(0,25$; $0,50$ e 0,75$)$.

Foi definido um intervalo de confiança de $95 \%$ ( $\mathrm{p}<0.05)$ para o teste de significância dos parâmetros isolados e suas interações e a análise múltipla de médias foi realizada, objetivando determinar a faixa de variação do parâmetro que apresenta a condição de atrito baixo, ou seja maior raio interno possível (representado por um valor negativo).

\section{Resultados}

A partir das dimensões do modelo virtual deformado, as curvas de calibração foram traçadas em planilha "Excel". No mesmo gráfico foram marcadas os valores relativos as dimensões dos corpos de prova deformados para comparação visando determinar o valor de atrito por comparação com as curvas calibradas. Os resultados da análise comparativa são mostrados na Fig. 7.

\section{Discussão dos Resultados}

Objetivando comparar duas qualidades de lubrificantes comerciais disponíveis e recomendados para o forjamento de ligas de alumínio foi realizada a análise de variância dos parâmetros do ensaio do anel, observando a sua influência sobre o aumento do raio interno (variável de resposta). A Fig. 8 esquematiza o método de análise adotado.

Um aumento significativo no raio interno do anel (valor negativo) vai indicar que o atrito é baixo, portanto, o lubrificante apresenta um desempenho satisfatório (redução do atrito interfacial). Os dados tabelados para o lubrificante "Deltaforge 31" e a respectiva tabela ANOVA podem ser observados na Tabela 1.

Observa-se, no teste da tabela ANOVA, que as influências dos parâmetros temperatura (A), velocidade de deformação (B) e deformação linear na altura (C) são bastante significativas. Em função destes resultados, partiu-se para a análise múltipla de médias objetivando concluir a influência dos parâmetros. Nesta análise, fixam-se 2 parâmetros em relação a um 3o parâmetro. Será aceita a hipótese de significância quando a diferença entre as médias for maior que o valor calculado de $3 . S_{x}$, onde $S_{x}$ é o desvio padrão das

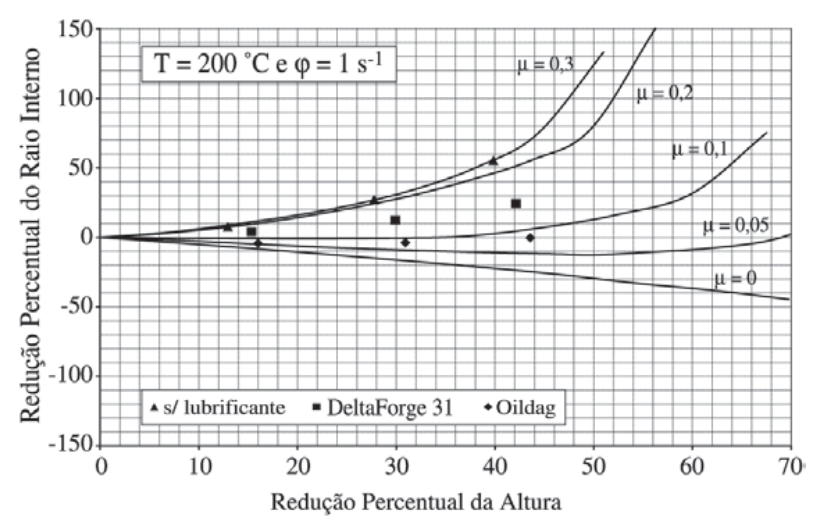

a)

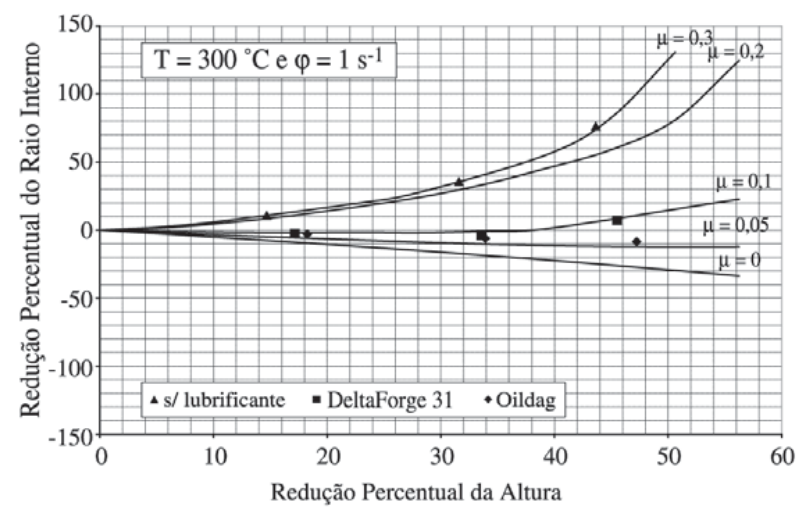

b)

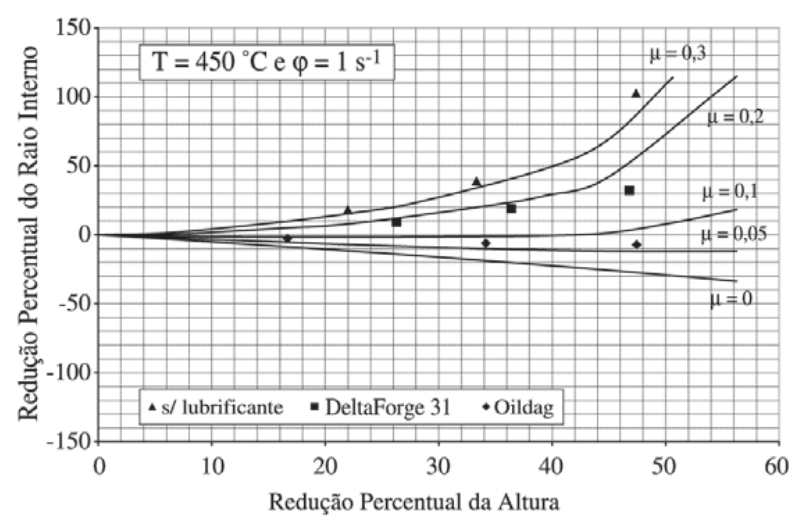

c)

Figura 7. Curvas de Calibração e Resultados de Atrito a) $\mathrm{T}=200$ ${ }^{\circ} \mathrm{C}, 1 \mathrm{~s}^{-1}$; b) $\mathrm{T}=300{ }^{\circ} \mathrm{C}, 1 \mathrm{~s}^{-1}$; c) $\mathrm{T}=450{ }^{\circ} \mathrm{C}, 1 \mathrm{~s}^{-1}$.

médias calculado por $\mathrm{S}_{\mathrm{x}}=\mathrm{SQRT}(\mathrm{MQR}) / \mathrm{SQRT}(\mathrm{n})$.

Desta comparação conclui-se que (a) para o lubrificante "Deltaforge 31" houve uma diminuição significativa do raio interno dos anéis deformados, ou seja, aumento do atrito na variação de temperatura de $200{ }^{\circ} \mathrm{C}$ até $450{ }^{\circ} \mathrm{C}$, deformação linear na altura 0,75 e velocidade de deformação $50 \mathrm{~s}^{-1} \mathrm{e}$ (b) 
Tabela 1. Dados e tabela ANOVA para o lubrificante "Deltaforge 31".

\begin{tabular}{|c|c|c|c|c|c|c|c|c|c|}
\hline \multicolumn{10}{|c|}{ Fator B - Velocidade de deformação } \\
\hline \multirow{3}{*}{$\begin{array}{l}\text { Fator A - } \\
\text { Temperatura }\end{array}$} & \multicolumn{3}{|c|}{$1 \mathrm{~s}^{-1}(\mathrm{~B} 1)$} & \multicolumn{3}{|c|}{$10 \mathrm{~s}^{-1}(\mathrm{~B} 2)$} & \multicolumn{3}{|c|}{$50 \mathrm{~s}^{-1}(\mathrm{~B} 3)$} \\
\hline & \multicolumn{9}{|c|}{ Fator C - Deformação Relativa } \\
\hline & $\varepsilon=0,25$ & $\varepsilon=0,50$ & $\varepsilon=0,75$ & $\varepsilon=0,25$ & $\varepsilon=0,50$ & $\varepsilon=0,75$ & $\varepsilon=0,25$ & $\varepsilon=0, .50$ & $\varepsilon=0,75$ \\
\hline & (C1) & $(\mathrm{C} 2)$ & (C3) & $(\mathrm{C} 1)$ & $(\mathrm{C} 2)$ & (C3) & $(\mathrm{C} 1)$ & $(\mathrm{C} 2)$ & (C3) \\
\hline \multirow{2}{*}{$\mathrm{T}=200^{\circ} \mathrm{C}(\mathrm{A} 1)$} & 3,73 & 12,27 & 24,22 & 4,01 & 11,99 & 26,43 & 5,12 & 19,50 & 43,17 \\
\hline & 3,73 & 12,27 & 24,22 & 4,01 & 11,99 & 26,43 & 5,12 & 19,50 & 43,17 \\
\hline \multirow[t]{2}{*}{$\mathrm{T}=300^{\circ} \mathrm{C}(\mathrm{A} 2)$} & $-2,60$ & $-4,24$ & 7,05 & 2,75 & 6,78 & 19,16 & 3,55 & 11,06 & 14,31 \\
\hline & $-2,60$ & $-4,24$ & 7,05 & 2,75 & 6,78 & 19,16 & 3,55 & 11,06 & 14,31 \\
\hline \multirow[t]{2}{*}{$\mathrm{T}=450{ }^{\circ} \mathrm{C}(\mathrm{A} 3)$} & 8,92 & 18,89 & 31,97 & 7,95 & 21,44 & 31,51 & 9,79 & 50,63 & 152,32 \\
\hline & 8,92 & 18,89 & 31,97 & 7,95 & 21,44 & 31,51 & 9,79 & 50,63 & 152,32 \\
\hline Variável & \multicolumn{2}{|c|}{ Soma de Quadrados } & \multicolumn{2}{|c|}{ GDL } & \multicolumn{2}{|c|}{ Médias Quadradas } & Teste F & \multicolumn{2}{|c|}{ F Tabelado } \\
\hline A & \multicolumn{2}{|r|}{4373,67} & \multicolumn{2}{|c|}{2} & \multicolumn{2}{|c|}{2186,84} & 9,82 & \multicolumn{2}{|r|}{4,46} \\
\hline B & \multicolumn{2}{|r|}{2826,07} & \multicolumn{2}{|c|}{2} & \multicolumn{2}{|c|}{1413,03} & 6,35 & \multicolumn{2}{|r|}{4,46} \\
\hline C & \multicolumn{2}{|r|}{5407,28} & \multicolumn{2}{|c|}{2} & \multicolumn{2}{|c|}{2703,64} & 12,15 & \multicolumn{2}{|r|}{4,46} \\
\hline $\mathrm{AB}$ & \multicolumn{2}{|r|}{2672,97} & \multicolumn{2}{|c|}{4} & \multicolumn{2}{|c|}{668,24} & 3,00 & \multicolumn{2}{|r|}{3,84} \\
\hline $\mathrm{AC}$ & \multicolumn{2}{|r|}{2113,90} & \multicolumn{2}{|c|}{4} & \multicolumn{2}{|c|}{528,47} & 2,37 & \multicolumn{2}{|r|}{3,84} \\
\hline BC & \multicolumn{2}{|r|}{3509,98} & & & 877 , & & 3,94 & & 3,84 \\
\hline $\mathrm{ABC}$ & & 1780,70 & & & 222 , & & 1,00 & & 3,44 \\
\hline Erro & & 0,00 & & & & & & & \\
\hline Total & & 2684,57 & & & & & & & \\
\hline
\end{tabular}

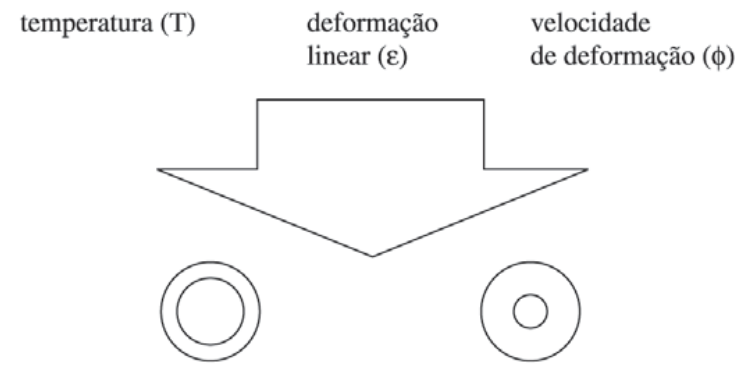

Atrito baixo $=$ raio interno maior $\quad$ Atrito alto $=$ raio interno menor

Figura 8. Esquema de análise do ensaio do anel.

para o lubrificante "Deltaforge 31" houve diminuição significativa do raio interno dos anéis deformados, ou seja, aumento do atrito quando da variação da deformação linear na altura de 0,50 até 0,75 , na temperatura de $450^{\circ} \mathrm{C}$ e velocidade de deformação $50 \mathrm{~s}^{-1}$.

Da mesma forma, Os dados tabelados para o lubrifican- te "Oildag" e a respectiva tabela ANOVA podem ser observados na Tabela 2.

Observa-se no teste da tabela ANOVA que as influências dos parâmetros temperatura $(\mathrm{A})$, velocidade de deformação (B) e deformação linear na altura (C) NÃO são significativas para o lubrificante "Oildag". A interação entre a velocidade de deformação e a deformação linear na altura apresenta sim, uma considerável significância.

$\mathrm{Na}$ análise múltipla de médias realizada da mesma forma que na tabela anterior, conclui-se que para o lubrificante "Oildag" houve aumento do raio interno dos anéis deformados, ou seja, redução do atrito quando das variações de temperatura, velocidade de deformação e deformação linear em altura estudadas nos ensaios.

\section{Conclusões}

Com base nas análises discutidas, conclui-se que o lubrificante "Oildag" apresenta uma maior estabilidade em relação ao lubrificante "Deltaforge 31" frente as variações de temperatura, deformação e velocidade de deformação na função de reduzir o atrito interfacial peça-ferramenta.

Adicionalmente à análise estatística, avaliando os lubri- 
Tabela 2. Dados e tabela ANOVA para o lubrificante "Oildag”.

\begin{tabular}{|c|c|c|c|c|c|c|c|c|c|}
\hline \multicolumn{10}{|c|}{ Fator B - Velocidade de deformação } \\
\hline \multirow{3}{*}{$\begin{array}{l}\text { Fator A - } \\
\text { Temperatura }\end{array}$} & \multicolumn{3}{|c|}{$1 \mathrm{~s}^{-1}(\mathrm{~B} 1)$} & \multicolumn{3}{|c|}{$10 \mathrm{~s}^{-1}(\mathrm{~B} 2)$} & \multicolumn{3}{|c|}{$50 \mathrm{~s}^{-1}(\mathrm{~B} 3)$} \\
\hline & \multicolumn{9}{|c|}{ Fator C - Deformação Relativa } \\
\hline & $\varepsilon=0,25$ & $\varepsilon=0,50$ & $\varepsilon=0,75$ & $\varepsilon=0,25$ & $\varepsilon=0,50$ & $\varepsilon=0,75$ & $\varepsilon=0,25$ & $\varepsilon=0,50$ & $\varepsilon=0,75$ \\
\hline \multirow{3}{*}{$\mathrm{T}=200^{\circ} \mathrm{C}(\mathrm{A} 1)$} & (C1) & $(\mathrm{C} 2)$ & (C3) & (C1) & $(\mathrm{C} 2)$ & (C3) & (C1) & $(\mathrm{C} 2)$ & (C3) \\
\hline & $-4,31$ & $-3,85$ & $-0,33$ & $-4,24$ & $-3,00$ & $-4,15$ & $-4,39$ & $-1,48$ & 1,44 \\
\hline & $-4,31$ & $-3,85$ & $-0,33$ & $-4,24$ & $-3,00$ & $-4,15$ & $-4,39$ & $-1,48$ & 1,44 \\
\hline \multirow[t]{2}{*}{$\mathrm{T}=300{ }^{\circ} \mathrm{C}(\mathrm{A} 2)$} & $-2,85$ & $-6,05$ & $-8,43$ & $-6,32$ & $-10,51$ & 11,27 & 15,22 & 12,74 & $-12,42$ \\
\hline & $-2,85$ & $-6,05$ & $-8,43$ & $-6,32$ & $-10,51$ & 11,27 & 15,22 & 12,74 & $-12,42$ \\
\hline \multirow[t]{2}{*}{$\mathrm{T}=450{ }^{\circ} \mathrm{C}(\mathrm{A} 3)$} & $-2,67$ & $-6,08$ & $-3,79$ & $-3,79$ & $-6,79$ & $-4,86$ & $-4,69$ & $-7,51$ & $-4,55$ \\
\hline & $-2,67$ & $-6,08$ & $-3,79$ & $-3,79$ & $-6,79$ & $-4,86$ & $-4,69$ & $-7,51$ & $-4,55$ \\
\hline Variável & \multicolumn{2}{|c|}{ Soma de Quadrados } & \multicolumn{2}{|c|}{ GDL } & \multicolumn{2}{|c|}{ Médias Quadradas } & Teste F & \multicolumn{2}{|c|}{ F Tabelado } \\
\hline A & \multicolumn{2}{|r|}{93,40} & \multicolumn{2}{|c|}{2} & \multicolumn{2}{|c|}{46,70} & 2,12 & \multicolumn{2}{|r|}{4,46} \\
\hline B & \multicolumn{2}{|r|}{78,18} & \multicolumn{2}{|c|}{2} & \multicolumn{2}{|c|}{39,09} & 1,78 & \multicolumn{2}{|r|}{4,46} \\
\hline $\mathrm{C}$ & \multicolumn{2}{|r|}{12,83} & \multicolumn{2}{|c|}{2} & \multicolumn{2}{|c|}{6,41} & 0,29 & \multicolumn{2}{|r|}{4,46} \\
\hline $\mathrm{AB}$ & \multicolumn{2}{|r|}{115,17} & \multicolumn{2}{|c|}{4} & \multicolumn{2}{|c|}{28,79} & 1,31 & \multicolumn{2}{|r|}{3,84} \\
\hline $\mathrm{AC}$ & \multicolumn{2}{|r|}{59,55} & \multicolumn{2}{|c|}{4} & \multicolumn{2}{|c|}{14,89} & 0,68 & \multicolumn{2}{|r|}{3,84} \\
\hline $\mathrm{BC}$ & \multicolumn{2}{|r|}{550,93} & & & 137, & & 6,26 & & 3,84 \\
\hline $\mathrm{ABC}$ & & 176,04 & & & 22,0 & & 1,00 & & 3,44 \\
\hline Erro & & 0,00 & & & & & & & \\
\hline Total & & 1086,10 & & & & & & & \\
\hline
\end{tabular}

ficantes quanto ao coeficiente de atrito plotado na comparação com as curvas de calibração (Fig. 7), pode-se observar que o lubrificante "Oildag" apresenta coeficientes menores $(0,05<\mu<0,1)$ que o lubrificante "Deltaforge 31".

Quanto às características ${ }^{2}$ abordadas por Altan (1983), o lubrificante "Oildag" peca em duas:

a) Durante o aquecimento do experimento foi sentido um forte cheiro de amônia;

b) Houve uma extrema dificuldade em se fazer a limpeza dos corpos de prova deformados no teste do "Oildag".

\section{Agradecimentos}

Os autores agradecem à Escola de Engenharia da UFRGS (LdTM), ao CNPq pelo apoio financeiro dos recursos do Banco Mundial, através do Edital III do PADCT, possibilitando a aquisição de materiais, equipamentos e bolsas de pesquisa e a Universidade Técnica de Aachen na Alemanha, na pessoa do Prof. Dr. Ing. Reiner Kopp pela doação da estação de trabalho e do programa de simulação computacional "Larstran".

\section{Referências}

1. Ribeiro, J.D. Avaliação do Atrito no Forjamento. dissertação de mestrado, Escola de Engenharia PPGEMM, Universidade Federal do Rio Grande do Sul, Porto Alegre, 1993.

2. Altan, T. et al. Metal Forming - Fundamentals and Applications, Ed. Carnes Publications Services, Inc., p. 8590, USA, 1983.

3. Schaeffer, L. Conformação do Metais., Ed. Rígel, p. 5659, Porto Alegre, 1995.

4. Avitzur, B.M. Metal Forming: Process and analysis, Ed. McGraw Hill Book Company, USA, 1968.

5. Marcon, A.F.; Bittencourt, E.; Creus, G.J. Single-test Determination of the Friction Coefficient and Stress-strain Curve for Application in Metal-forming Analyses, In: Experimental Mechanichs, Society for Experimental Mechanichs, v. 40, p. 124-125, USA, 2000.

6. Kopp, R.; Wiegels, H. Einführung in Die Umformtechnik, Verlag der Angustinus Buchhadlung, Alemanha, p. 6574, 1998. 